\title{
Initial Results of Hydrogen and Deuterium Beam Ion Simultaneous Transport due to Toroidal Alfvén Eigenmode in the Large Helical Device*)
}

\author{
Shuji KAMIO ${ }^{1)}$, Yutaka FUJIWARA ${ }^{1)}$, Kunihiro OGAWA ${ }^{1,2)}$, Ryosuke SEKI ${ }^{1,2)}$, \\ Kenichi NAGAOKA ${ }^{1,3)}$, Hideo NUGA ${ }^{1)}$, Siriyaporn SANGAROON ${ }^{1,4)}$, Mitsutaka ISOBE ${ }^{1,2)}$, \\ Masaki OSAKABE ${ }^{1,2)}$, Chio-Zong $\mathrm{CHENG}^{5,6)}$ and the LHD Experiment Group \\ ${ }^{1)}$ National Institute for Fusion Science, National Institute of Natural Sciences, 322-6 Oroshi-cho, Toki 509-5292, Japan \\ ${ }^{2)}$ The Graduate University for Advanced Studies, SOKENDAI, 322-6 Oroshi-cho, Toki 509-5292, Japan \\ ${ }^{3)}$ Nagoya University, Furo-cho, Chikusa-ku, Nagoya 464-8603, Japan \\ ${ }^{4)}$ Mahasarakham University, 20-41 Kamriang, Kantharawichai, Mahasarakham 44150, Thailand \\ ${ }^{5)}$ The University of Tokyo, 5-1-5 Kashiwanoha, Kashiwa 277-8561, Japan \\ ${ }^{6)}$ Princeton University, Princeton, New Jersey 08543, USA
}

(Received 8 December 2020 / Accepted 9 February 2021)

\begin{abstract}
The behavior of energetic particles (EPs) associated with toroidal Alfvén eigenmode (TAE) activities during the combined injection of hydrogen and deuterium beams was investigated in the Large Helical Device (LHD). The enhanced transports of both proton and deuteron with TAE activities were simultaneously observed by a tangentially viewing and mass and energy resolved neutral particle analyzer (E||B-NPA). At the timing of the TAE bursts with the mode number $n=1$, both proton and deuteron were transported to the outboard and observed with the similar energies of $137-138 \mathrm{keV}$. At the peak amplitude of the magnetic fluctuations measured by the Mirnov coils, the mixed frequencies of $64 \mathrm{kHz}$ and $29 \mathrm{kHz}$ were identified, and the observed frequencies did not chirp down. The observed timings of the transported hydrogen and deuterium were just after the magnetic fluctuations of $64 \mathrm{kHz}$ and $29 \mathrm{kHz}$, respectively. By adapting the cross-correlation analysis, the delay times from the magnetic fluctuation to the detection of EPs by E\|B-NPA are estimated to $95 \mu$ s and $145 \mu$ s for hydrogen and deuterium, respectively. These delays are considered to be the time of the radial transport, and the time delays depended on the velocities of the transported EPs.
\end{abstract}

(C) 2021 The Japan Society of Plasma Science and Nuclear Fusion Research

Keywords: toroidal Alfvén eigenmode, TAE burst, neutral particle analyzer

DOI: $10.1585 /$ pfr. 16.2402044

\section{Introduction}

In the research for the magnetic confinement fusion plasma, the study of the toroidal Alfvén eigenmode (TAE) [1] is one of the important topic for the future fusion reactor. The TAE instability can be caused by the energetic particles (EPs) generated by fusion reaction and/or by external source of heating/current-drive. The excited TAE might deteriorate the confinement performance of the EPs by transport of the resonant EPs, and cause the damage of the first wall by the lost EPs. In order to understand the behavior of the TAE and its interaction with EPs, the particle behavior caused by TAE has been studied in many experimental devices [2-4]. Also in the Large Helical Device (LHD), the TAE activities were observed and investigated in the relatively low magnetic field experiment with the tangentially injected neutral beams (NBs) [5-8].

In the recent study of the bursting TAE in LHD, the

author'se-mail: kamio@nifs.ac.jp

*) This article is based on the presentation at the 29th International Toki Conference on Plasma and Fusion Research (ITC29). transported EPs due to the TAE activities were observed using a mass and energy resolved E-parallel-B-type neutral particle analyzer (E\|B-NPA) $[9,10]$, and investigated in detail in the experimental condition of using pure hydrogen for the injection beams and bulk plasma [8]. In the TAE burst experiment with pure hydrogen, the frequency of the magnetic fluctuations of the TAE burst with the toroidal mode number $n=1$ were chirping down from $75 \mathrm{kHz}$ to $50 \mathrm{kHz}$. The observed particle energy which can be considered to be the energy of the resonant particles was $150 \mathrm{keV}$ to $90 \mathrm{keV}$. The relationship of the magnetic fluctuation frequency chirping and the energy slowing down of the observed transported EPs during TAE bursts were well explained.

On the other hand, in the future fusion reactor, TAE might be induced by two different EP species, i.e., energetic alpha particles produced by fusion reaction and energetic deuterons introduced as an external current drive source. Therefore, it is also important to study the TAE activities in the condition with existing the different EP 
species. Using the benefit of high energy NB injectors on LHD, we can simulate the different EP species condition by simultaneous hydrogen and deuterium NB injection.

\section{Experimental Setup}

The toroidal cross-section view of LHD and the directions of the NBs are shown in Fig. 1. Three tangential NBs were used for the bursting TAE experiment. In order to simulate the condition of the different species of EPs, NB \#1 and NB \#2 were used for injecting deuterium NB, and NB \#3 were used for injecting hydrogen NB in the deuterium plasma. The NBs are also used for the plasma start-up in the relatively low magnetic field experiments in LHD [11].

In order to observe the transported EPs due to the bursting TAE, E\|B-NPA were used for observing the transported hydrogen and deuterium simultaneously. E||B-NPA measures the EPs which are charge exchanged with the neutral particles at the outer region. Therefore, the increase of the EPs flux observed by E||B-NPA at the TAE burst can be considered to be the transported EPs to the outer region. E||B-NPA can measure the both hydrogen and deuterium at a same time with the time resolution of $10 \mu \mathrm{s}$. As shown the line of sight of the E\|B-NPA in Fig. 1, E\|B-NPA measures the EPs injected by NB \#1 and \#3. From the result of the previous study [8], the transported energetic ions are considered to be charge exchanged at around $\rho_{\mathrm{LOS}}=0.98$ 1.02 at the TAE burst. Here, $\rho_{\text {LOS }}$ is the measurement region along the line of sight of the E||B-NPA. The length from the $\rho_{\text {LOS }}$ to the micro channel plate (MCP) inside the E\||B-NPA is approximately $8.5 \mathrm{~m}$.

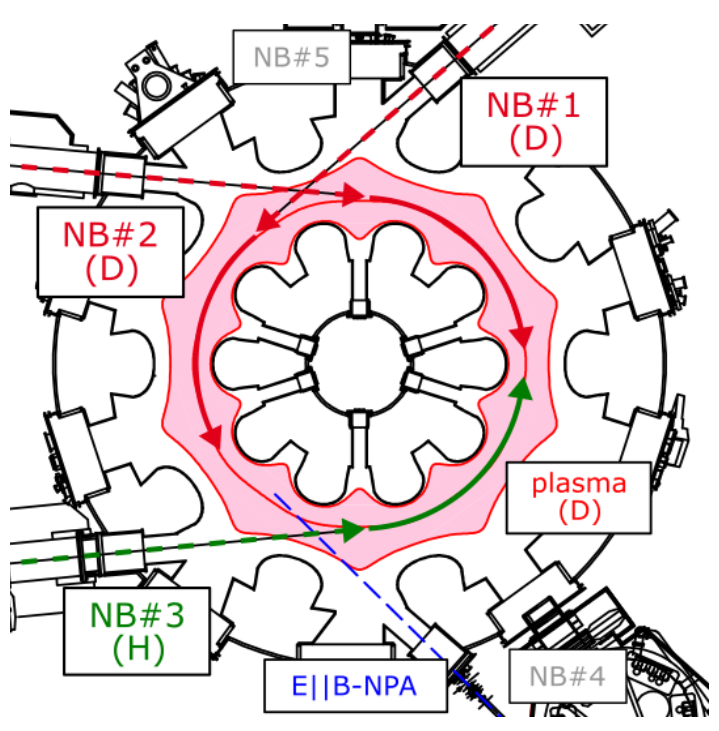

Fig. 1 Chematic view of the LHD, the directions of the NBs, and the line of sight of the E $\mid$ B-NPA. E $\mid$ B-NPA can measure the EPs injected by NB \#1 and NB \#3.

\section{Experimental Results}

The TAE activities are successfully produced by the mixed energetic ion species of hydrogen and deuterium. Figure 2 shows the time evolutions of the parameters of the TAE burst experiment. The injection energies and powers of the NBs are shown in Figs. 2 (a) and (b). The injection energies of NB \#1 and NB \#3 are $153 \mathrm{keV}$ and $163 \mathrm{keV}$, respectively. When the Alfvén velocity $v_{\mathrm{A}}$ is $2,900 \mathrm{~km} / \mathrm{s}$ with the magnetic field of $B=0.6 \mathrm{~T}$ and the deuteron density of $n_{\mathrm{i}}=1 \times 10^{19} \mathrm{~m}^{-3}$, the beam velocity ratios are $v_{\text {Hbeam }} / v_{\mathrm{A}}=1.7$ and $v_{\text {Dbeam }} / v_{\mathrm{A}}=1.2$. The timing of the TAE activities was at approximately $4.2-4.8 \mathrm{~s}$. In the deuterium experiment, the neutron emission rate $S_{\mathrm{n}}$ can be measured by fission chamber [12] as shown in Fig. 2 (c). Similar to the time evolutions of the stored energy $W_{\mathrm{p}}$, electron temperature $T_{\mathrm{e}}$ and density $n_{\mathrm{e}}$, shown in Figs. 2 (d) and (e), significant difference between with and without TAE bursts does not be observed also on $S_{\mathrm{n}}$. These results indicate that a small part of the EPs were transported due to the TAE. The spatial distributions of the $T_{\mathrm{e}}$ and the $n_{\mathrm{e}}$ measured by Thomson scattering are shown in Fig. 3 (a). The spatial distributions of the electron temperature and density is similar to the previous TAE burst experiment in the hydrogen plasma [8]. Figure 3 (b) shows the shear Alfvén

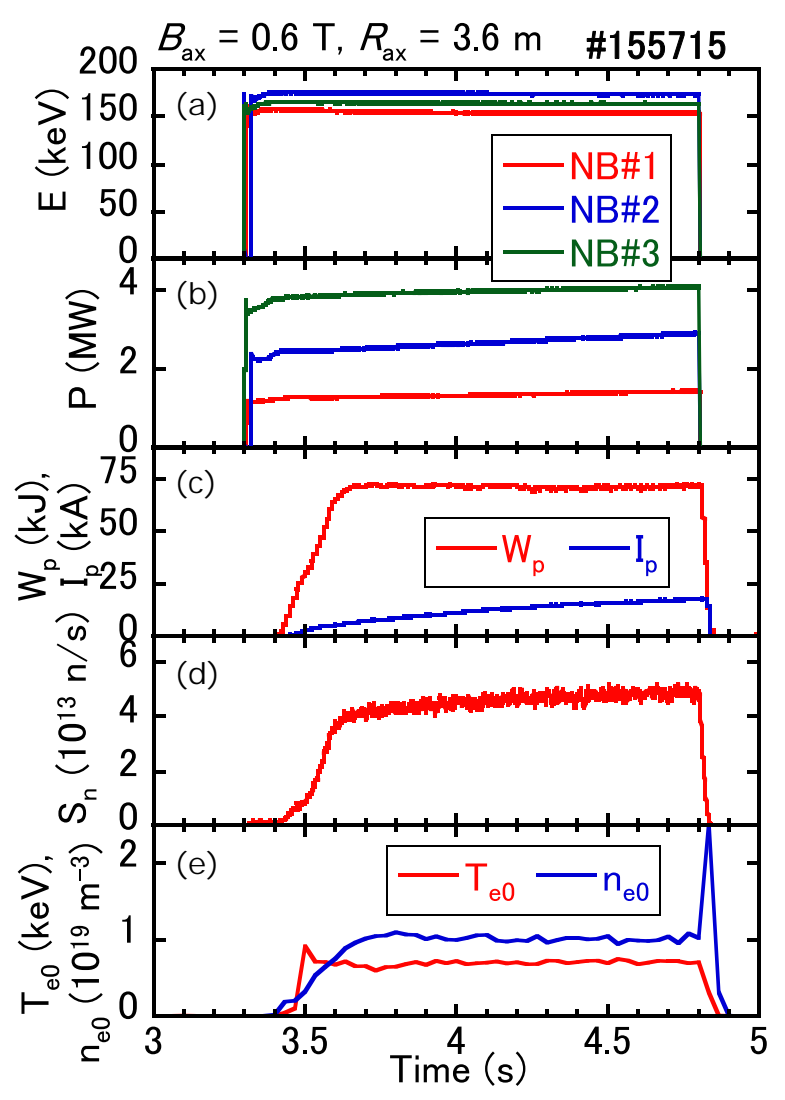

Fig. 2 Time evolutions of the (a) injection energies and (b) injection powers of NBs, (c) the stored energy and the plasma current, (d) the neutron emission rate, (e) the electron density and the temperature at the magnetic axis. 

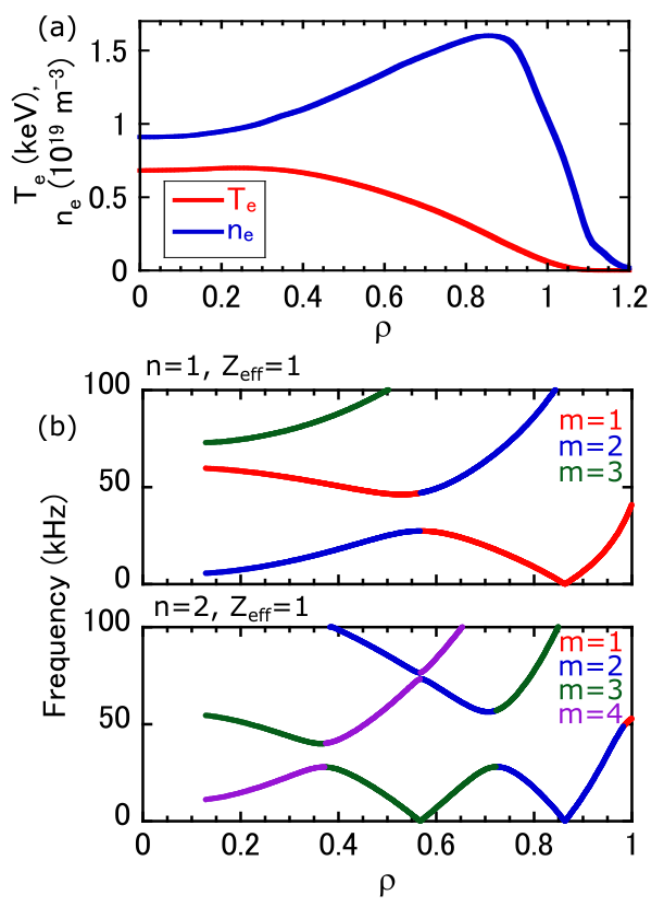

Fig. 3 (a) Spatial distributions of $T_{\mathrm{e}}$ and $n_{\mathrm{e}}$ during the TAE bursts. (b) The shear Alfvén continua with $n=1$ and $n=2$, calculated by STELLGAP code.

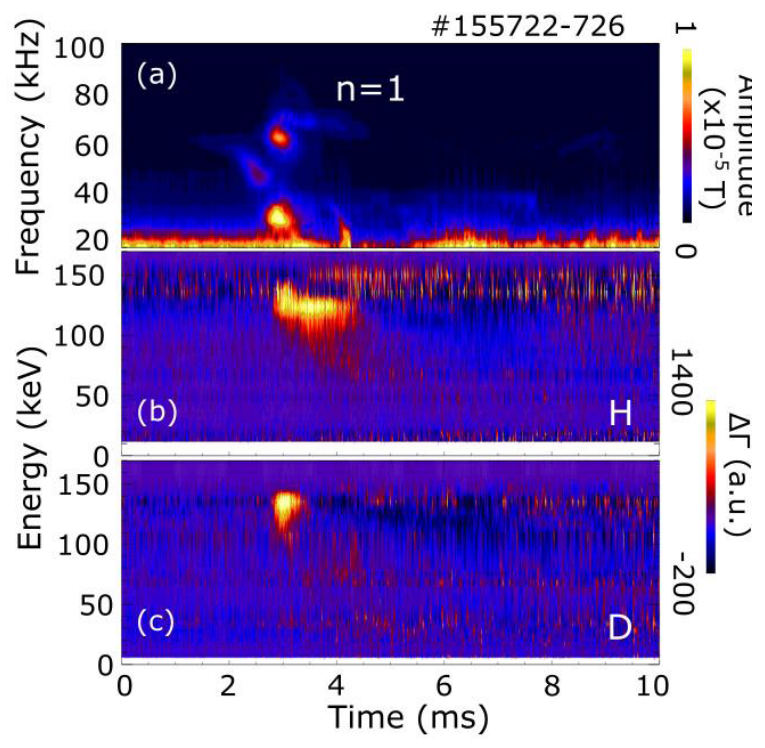

Fig. 4 Conditional averaged spectrogram of (a) the magnetic fluctuation $\tilde{b}$ and (b), (c) the change of EP flux energy spectra $\Delta \Gamma$ observed by E $\| \mathrm{B}-\mathrm{NPA}$ hydrogen and deuterium channels.

spectra with $n=1$, calculated by STELLGAP code [13]. The TAE $n=1$ mode gap is appeared at around $\rho=0.5$ 0.6 with the frequency of $30-50 \mathrm{kHz}$.

Figure 4 (a) shows the conditional averaged spectrogram of the magnetic fluctuation with the peak amplitude of $60 \mathrm{kHz}$ set to $3.0 \mathrm{~ms}$ for the conditional average. As shown in Fig. 4 (a), during the TAE burst experiment, two

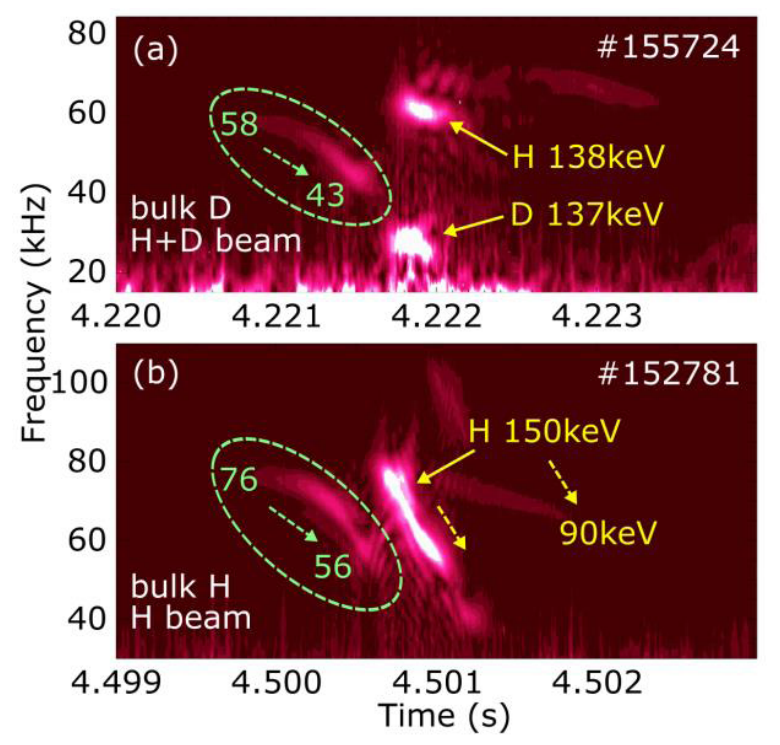

Fig. 5 Spectrograms of the magnetic fluctuations $\tilde{b}$ in the (a) hydrogen and deuterium mixed beam experiment and in the (b) pure hydrogen experiment.

kinds of bursts were observed by Mirnov coils. One is the $n=1$ mode with the mixture of several frequencies, and the other is the $n=2$ mode with the frequency chirping. This paper focuses on the $n=1$ mode because the $n=1$ mode was also observed in the previous study using the hydrogen NBs and the hydrogen bulk plasma [8]. Figures 4 (b), (c) show the energy spectra of the increase of EP flux $\Delta \Gamma$ observed by E $\| \mathrm{B}-\mathrm{NPA}$ hydrogen and deuterium channels. At the timing of the TAE bursts with the mode number $n=1$, both proton and deuteron were transported to the outboard and observed with the similar energies of $137-138 \mathrm{keV}$. At the peak amplitude of the magnetic fluctuations, the mixed frequencies of $64 \mathrm{kHz}$ and $29 \mathrm{kHz}$ were identified, and the observed frequencies did not chirp down. The observed timings of the transported hydrogen and deuterium were after the magnetic fluctuations of $64 \mathrm{kHz}$ and $29 \mathrm{kHz}$, respectively. However, by considering the resonance frequency $f_{\text {TAE }}$ given by $f_{\mathrm{TAE}}=v_{0} \cos \theta /\left(4 \pi q_{\mathrm{TAE}} R\right)$, frequency of $29 \mathrm{kHz}$ is too low as the resonance frequency with the $137 \mathrm{keV}$ deuteron by TAE. The reason for these relationships are under discussion. Here, $v_{0}$ is the velocity of the resonance particle, $\theta$ is the pitch angle of the resonance particle, $q_{\mathrm{TAE}}=$ $(m+1 / 2) / n=3 / 2$, and $R$ is the major radius.

As shown with green dashed circles in the Fig. 5 in both TAE burst experiment with mixed NB case and pure hydrogen NB case, the frequency chirping down of the magnetic fluctuations were observed before the peak amplitude of the magnetic fluctuations. The frequencies were $58 \mathrm{kHz}$ to $43 \mathrm{kHz}$ in the mixed NB and deuterium bulk plasma case, and $76 \mathrm{kHz}$ to $56 \mathrm{kHz}$ in the hydrogen NB and hydrogen bulk plasma case. Because the Alfvén velocity $v_{\mathrm{A}}$ is inversely proportional to the ion mass $m_{\mathrm{i}}$ with 


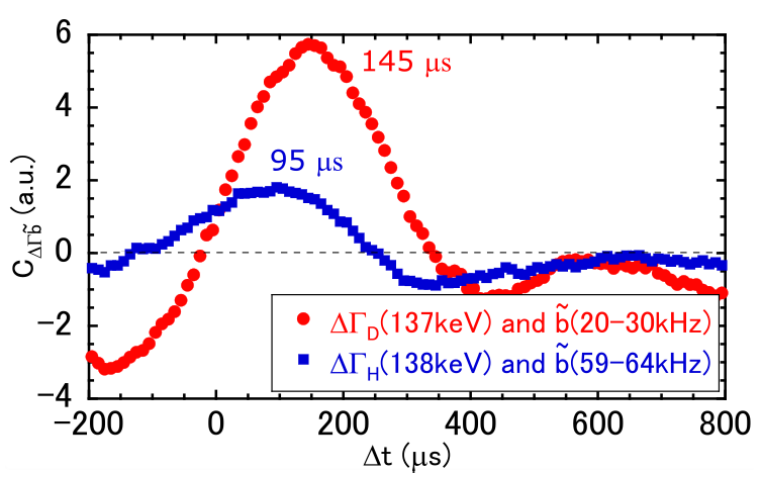

Fig. 6 The cross-correlation function $C_{\Delta \Gamma \tilde{b}}$ of $137 \mathrm{keV} \Delta \Gamma_{\mathrm{D}}$ and $\tilde{b} 20-30 \mathrm{kHz}$ (red), and the $C_{\Delta \Gamma \tilde{b}}$ of $138 \mathrm{keV} \Delta \Gamma_{\mathrm{H}}$ and $\tilde{b}$ $59-64 \mathrm{kHz}$ (blue).

$1 / \sqrt{m_{i}}$, this difference among the frequencies is reasonable. On the other hand, in the mixed NB case, the main fluctuation appeared at approximately $30 \mathrm{kHz}$ and $60 \mathrm{kHz}$, and the frequencies do not chirp down.

In the mixed NB case, the main bursts are not chirped down. Therefore, the time delay from the magnetic fluctuations of $\tilde{b}$ to observe the transported EPs can be estimated. In order to estimate the time delay $\Delta t$, cross-correlation analysis is adapted. The cross-correlation for the observed transported EP flux $\Delta \Gamma$ and magnetic fluctuation $\tilde{b}$ can be expressed with the time delay $\Delta t$ as

$$
C_{\Delta \Gamma \tilde{b}}(\Delta t)=\sum_{t} \Delta \Gamma(t+\Delta t) \tilde{b}(t)-\mu_{\Delta \Gamma} \mu_{\tilde{b}},
$$

where $\mu_{\Delta \Gamma}$ and $\mu_{\tilde{b}}$ are the average of $\Delta \Gamma$ and $\tilde{b}$. As shown in Fig. 6, the cross-correlation function of $\Delta \Gamma_{\mathrm{D}}(137 \mathrm{keV})$ and $\tilde{b}(20-30 \mathrm{kHz})$ is maximized with $\Delta t=145 \mu \mathrm{s}$, and the cross-correlation function of $\Delta \Gamma_{\mathrm{H}}(138 \mathrm{keV})$ and $\tilde{b}(59$ $64 \mathrm{kHz}$ ) is maximized with $\Delta t=95 \mu \mathrm{s}$. These delays can be considered to be the time of the radial transport after the resonant with the TAE. Because the delay of the $\Delta \Gamma$ from $\tilde{b}$ depends on the particle mass, the radial transport is related to the velocity of the transported EPs. The distances traveled during the delay time of $145 \mu$ s for $137 \mathrm{keV}$ proton and $95 \mu$ s for $138 \mathrm{keV}$ deuteron are approximately 500 meters in both cases.

\section{Summary}

The behavior of EPs associated with TAE activities is investigated in detail by a mass and energy resolved E $\mid \mathrm{B}$ NPA in LHD. During the combined injection of hydrogen and deuterium NBs, the enhanced transport of both proton and deuteron with TAE activities were simultaneously observed for the first time. Two different mode activities with the toroidal numbers of $n=1$ and $n=2$ were observed. Before the peak amplitude of the magnetic fluctuations of $n=1$ mode, the frequency chirping down from $58 \mathrm{kHz}$ to $43 \mathrm{kHz}$ was observed without the detection of EPs by E\|B-NPA. This initial burst seems to be similar to the TAE burst experiment with pure hydrogen case of $76 \mathrm{kHz}$ to $56 \mathrm{kHz}$. The peak amplitudes of the $n=1$ mode bursts were observed at around $30 \mathrm{kHz}$ and $60 \mathrm{kHz}$ at the similar timing. During the mode activities, the clear interaction of EPs with these modes are observed by the E\|BNPA both at the hydrogen and the deuterium channels at the similar energies of $137-138 \mathrm{keV}$, simultaneously. The transported hydrogen was observed during the magnetic fluctuation of $60 \mathrm{kHz}$, and the transported deuterium was observed during the magnetic fluctuation of $30 \mathrm{kHz}$. By the cross-correlation analysis, the delays from the magnetic fluctuations $\tilde{b}$ to the detection of the EPs by E||B-NPA are estimated to be $95 \mu$ s and $145 \mu$ s, respectively.

\section{Acknowledgements}

This work is supported by NIFS Grant (ULRR006, ULRR035, and ULRR702).

[1] C.Z. Cheng and M.S. Chance, Phys. Fluids 29, 3695 (1986).

[2] W.W. Heidbrink et al., Nucl. Fusion 31, 1635 (1991).

[3] D.S. Darrow et al., Nucl. Fusion 37, 939 (1997).

[4] M.P. Gryaznevich and S.E. Sharapov, Nucl. Fusion 46, S942 (2006).

[5] K. Toi et al., Plasma Phys. Control. Fusion 46, S1 (2004).

[6] K. Ogawa et al., Nucl. Fusion 50, 084005 (2010).

[7] M. Osakabe et al., Nucl. Fusion 46, S911 (2006).

[8] S. Kamio et al., Nucl. Fusion 60, 112002 (2020).

[9] S.S. Medley and A.L. Roquemore, Rev. Sci. Instrum. 69, 2651 (1998).

[10] Y. Fujiwara et al., JINST 15, C02021 (2020).

[11] O. Kaneko et al., Nucl. Fusion 39, 1087 (1999).

[12] M. Isobe et al., Rev. Sci. Instrum. 85, 11E114 (2014).

[13] D.A. Spong et al., Phys. Plasmas 17, 022106 (2010). 